\title{
Frontal plane tibio-femoral alignment and its association with knee pain among patients with early osteoarthritis of the knee in a population presenting to a tertiary hospital in South India
}

\author{
M. Koteshwar Rao, P. Lakshmi Narayana Reddy*
}

Orthopedic Speciality Hospital, Pogathota, Nellore, Andhra Pradesh, India

Received: 21 September 2017

Revised: 07 November 2017

Accepted: 08 November 2017

\section{*Correspondence:}

Dr. P. Lakshmi Narayana Reddy,

E-mail: lakshminarayana.ortho@rediffmail.com

Copyright: () the author(s), publisher and licensee Medip Academy. This is an open-access article distributed under the terms of the Creative Commons Attribution Non-Commercial License, which permits unrestricted non-commercial use, distribution, and reproduction in any medium, provided the original work is properly cited.

\begin{abstract}
Background: Frontal plane knee malalignment may increase the progression of knee osteoarthritis (OA) and worsen functional capacity.

Methods: In our study, the aim was to find the correlation of frontal plane tibiofemoral alignment with knee pain in early OA knee patients. After meeting the inclusion and exclusion criteria, 195 patients with 314 knees (104 females, 91 males) were studied. Clinical and radiological measurements of frontal plane tibiofemoral alignment, using landmark of hip, knee, and ankle, compared with knee pain.

Results: The accepted Statistical significance was $\mathrm{p}<0.05$. Patients with 130 knees $(41 \%)$ were normal alignment, and 184 knees $(59 \%)$ were abnormal alignment. The mean \pm SD age in our study was $54.6 \pm 8.9$ years. The mean \pm SD BMI in our study to be $25.86 \pm 1.96$. Mean \pm SD Q-angle in patients with normal and abnormal alignment was $14.76 \pm 1.54$ and $14.63 \pm 1.9$ respectively. There is statistically no significant correlation between BMI and VAS score $(r=0.054$, $\mathrm{p}=0.344$ ). Means \pm SD of mLDFA, mMPTA, mTFA, aTFA, and JOA were $88.86 \pm 2.73,84.96 \pm 3.00,5.96 \pm 4.01$, $4.55 \pm 3.67$, and $2.37 \pm 1.88$ respectively. JOA and mTFA had statistically significant weak positive correlation with VAS (knee pain) score, $(\mathrm{r}=0.281)(\mathrm{p}=0.000)$ and $(\mathrm{r}=0.236)(\mathrm{p}=0.000)$ respectively. The rest of the angles were not had statistically significant correlation with VAS score. The mTFA had statistically significant weak positive correlation with all the angles. We found that Frontal plane tibiofemoral alignment correlates with knee pain in patients with early OA.

Conclusions: The mTFA may be considered one important criterion in designing treatment and planning surgery for patients with primary osteoarthritis. The Q angle acts as a good alternative clinical tool in assessing frontal plane alignment. BMI was not correlating with knee pain (VAS score).
\end{abstract}

Keywords: Frontal plane tibio-femoral alignment, Osteoarthritis, VAS score

\section{INTRODUCTION}

Osteoarthritis (OA) is a degenerative disease of joint characterized by the breakdown of the articular cartilage and imbalance between its regeneration and repair. Osteoarthritis (OA) is a disabling disease affecting approximately $20 \%$ of the adult population. ${ }^{2}$ It is a significant cause of pain and disability in our aging population. Primary OA is known to affect the hip and knee. In the Asian population, the prevalence of osteoarthritis of the knee is known to be higher than that of the hip. This high prevalence in Asian population is presumed to be because of kneeling activities and floor activities being more compared to the western population. 
In Asian population, we have observed that osteoarthritis presents early and is progressive to require replacement at an earlier age. ${ }^{3}$

Patient characteristics that can predict progression of knee OA are age, presence of OA in multiple joints and alignment of the knee. Radiographic features like joint space narrowing (JSN), chondrocalcinosis, and severity of $\mathrm{OA}$ as measured by Kellegren Lawrence $(\mathrm{K} / \mathrm{L})$ grade and osteophyte score are also predictors of OA. Lower limb alignment is one of the predictors of the progression of knee OA. ${ }^{4}$ Recently a study has shown an association between baseline static knee angle and rate of cartilage loss in patients with knee OA. ${ }^{2}$ Cartilage defects in the knee, are recognized as important early determinants of potential arthritic change. ${ }^{5}$ Sharma et al demonstrated an association between knee malalignment and progression of uni-compartmental joint space narrowing. ${ }^{6}$ Varus alignment increases the risk of medial knee OA progression, and valgus alignment increases the risk of subsequent lateral knee OA progression. The severity of malalignment is directly proportional to the loss of joint space, and the resultant decline in the physical function.

Evaluation of knee alignment is useful for the diagnosis of arthritis and surgical planning. ${ }^{6}$ It can also serve as a guide for surgical planning. Radiographic frontal plane knee malalignment causes altered weight bearing stresses across the cartilage. This malalignment may predispose to early and rapid development of degenerative osteoarthritis. ${ }^{9}$ Frontal plane knee alignment measured radiographically indicates both presence and severity of knee osteoarthritis OA. It is prognostic of future worsening in persons with knee OA. ${ }^{6,7}$ Frontal plane alignment also is frequently considered during surgical and non-surgical approaches to treating knee pain.

The range of values for the Asian/Indian population has not been described separately. Abnormal load distribution across the joint can be influenced by minor alterations in knee alignment. A 4-6\% increase in varus alignment has been reported to increase loading in the medial compartment by up to $20 \%$. The increase in compartment loading that is thought to increase stress on articular cartilage and other joint structures leads to degenerative changes. ${ }^{8,9}$

Mechanical alignment measurements of the hip-kneeankle (HKA) angle from whole limb radiographs is superior to all other forms of radiographic measurement of frontal plane alignment. ${ }^{10,11}$ Malalignment of the lower extremity in the frontal plane like valgus and varus is a risk factor for the onset and progression of OA knee. ${ }^{12}$ These values are not defined for our population which sees early onset and greater severity of degenerative osteoarthritis. There is no literature studying that the effect of frontal tibiofemoral malalignment on osteoarthritis in Indian sub-continent and few studies are available on the relationship of tibiofemoral alignment and osteoarthritis.
It is worthwhile to know the presence of radiological malalignment of the lower extremities in individuals with early degenerative knee pain individuals to be able to correlate the onset of knee pain to the malalignment. ${ }^{13}$ This study aims to evaluate the frontal plane alignment around the knee in patients who are presenting with early onset of degenerative knee pain in an attempt to understand the prevalence of malalignment, and the possible correlation of clinical and radiological malalignment to knee pain in the population under study.

Sufficient study data for Indian or Asian Population for these parameters is not available. The knowledge of the normal frontal plane tibiofemoral can influence the planning of replacements as well as deformity corrections around the knee. Early detection can allow steps to prevent progression with less extensive options like osteotomy or braces.

Present study aimed to look for a possible correlation between frontal plane alignment and knee pain.

\section{METHODS}

This cross sectional study was conducted from July 2014 to July 2016 in the department of orthopedics JIPMER, Pondicherry. The study was approved by Institutional scientific committee and ethics committee. Patients presenting with degenerative knee pain as defined by American Rheumatology Association were evaluated for their knee pain. A detailed history and examination of the patients were done before including them into the study. Standard anteroposterior (weight bearing) and lateral radiographs of the knee were obtained for the patients with degenerative knee pain. The degenerative knee pain of the arthritic knee defined by the American College of Rheumatology Association (1986) is; Knee pain and osteophytes on radiographs; or Knee pain and patient's age of 40 years or more with morning stiffness lasting 30 minutes or less and crepitus on the motion. ${ }^{14}$

\section{Inclusion criteria}

Inclusion criteria were patients presenting to the OPD with knee pain of degenerative type and meeting the American College of Rheumatology criteria for the classification of OA of the knee. ${ }^{15,16}$ Radiographs showing Kellegren-Lawrence grades 1, 2 OA on anteroposterior view; patients having complete or near complete range of motion.

\section{Exclusion criteria}

Exclusion criteria were knee pain secondary to all other causes of arthritis like rheumatoid arthritis, secondary osteoarthritis, neoplasia and trauma; Radiographs showing greater than stage 2 Kellegren-Lawrence i.e., stages 3,4 OA on anteroposterior view; pre-existing bilateral congenital or developmental deformities like genu varum or valgum; presence of fixed deformities of knee; pre-existing hip or ankle deformities 


\section{Kellegren -Lawrence ${ }^{17}$}

Grade I: Unlikely narrowing of the joint space, possible osteophyte

Grade II: Small osteophytes, possible narrowing of the jointspace

Grade III: Multiple, moderately sized osteophytes, definite joint space narrowing, some sclerotic areas, possible deformation of bone ends

Grade IV: Multiple large osteophytes, severe joint space narrowing, marked sclerosis and definite bony end deformity.

The sample size was calculated to be 190. After meeting the inclusion criteria and exclusion criteria 195 patients who met the inclusion criteria were included into the study. The demographic and anthropometric data were obtained from all the patients. Severity of knee pain was measured on a visual analog scale (VAS).

\section{Q angle}

The Q-angle was measured by a hand-held goniometer, with the patient in supine position. The hip was kept in extension, neutral rotation, and the knee in complete extension. The feet were in the neutral position. The Q- angle was made by intersecting two lines at the center of the patella. The first line was drawn from the antero superior iliac spine (ASIS) to the center of the patella and the second line was drawn from the tibial tuberosity to the center of the patella. ${ }^{20}$ The intra and inter-observer reliability after the measurement of the Q-angle using a goniometer are well established across published studies. $^{21}$

After that, a standard standing long leg hip knee ankle radiograph obtained as mentioned below.

\section{Imaging}

Antero-posterior lower limb long radiographs (scanograms) imaging femur, tibia, ankle joints and feet were obtained in weight bearing position. Images were archived through PACS (picture archiving communication system) (GE Centricity universal viewer zero footprint Version: 6.0 SP1.1) and retrieved on the computer for analysis.

\section{Measurement of frontal plane knee alignment}

Centre of the femoral head was drawn by joining two points from center to periphery drawing radius equally as shown in Figure 1. The centre of the knee joint was made at the apex of the femoral notch. The Centre of the ankle joint was marked by the Centre of the talus Figure 1.

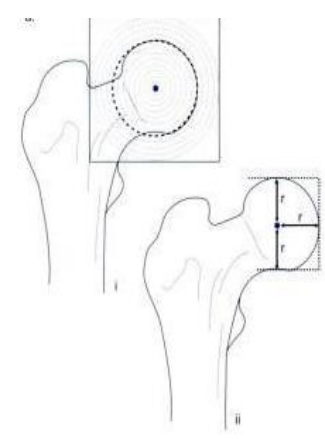

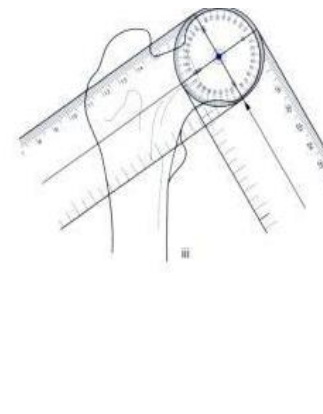

A

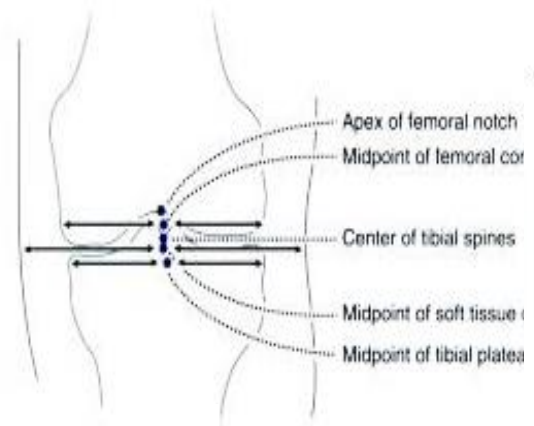

B

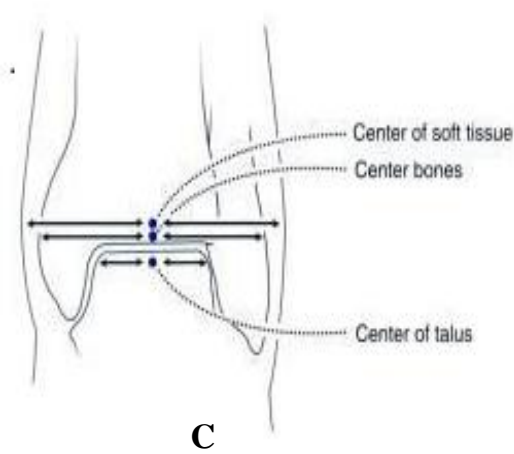

Figure 1: $A=$ centre of femoral head, $B=$ centre of knee, $C=$ centre of ankle.

HKA (mechanical) axis: The mechanical axis of the femur (FM) is as a line drawn from the center of the femoral head to the Centre of the knee joint. The mechanical axis of tibia (TM) is a line drawn from the center of the knee joint to the Centre of the talus.

Mechanical tibio-femoral angle: Angle between mechanical axes of femur and tibia.

m MDFA (mechanical medial distal femoral angle): Angle between mechanical axis of femur and femoral joint orientation line medially. m LDFA (mechanical lateral distal femoral angle): Angle between mechanical axis of femur and femoral joint orientation line laterally.

m LPTA (mechanical lateral proximal tibial angle): Angle between mechanical axis of tibia and tibial joint orientation line laterally.

M MPTA (mechanical medial proximal tibial angle): Angle between mechanical axis of tibia and tibial joint orientation line laterally. 
Anatomical tibio-femoral angle: Angle between anatomical axes of femur and tibia.

Joint orientation angle: Angle between femoral and tibial joint lines.

The distribution of data on demographic variables like age, gender, BMI status was expressed as frequency and percentages. The data on all continuous variables such as tibio-femoral angle, Q-Angle etc. was expressed as mean with standard deviation. The linear relationship between measures the frontal plane tibio-femoral alignment and osteoarthritic knee pain was carried out by using correlation analysis. All statistical analysis was done keeping 5 percent level of significance and p-value was $<0.05$ considered as significant.

\section{Significant Figures}

* Significant ( $\mathrm{P}$ value: $0.05<\mathrm{P}<0.10)$

** Moderate significant- (P value of 0.01 to 0.05 )

$* * *$ Strong significant $(\mathrm{P}$ value of $<0.01)$

*Weak correlation $(\mathrm{r}=0)$

**Moderate correlation $(\mathrm{r}=0.5$ to 0.8$)$

$* * *$ Strong correlation $(=-1$ or +1$)$.

\section{RESULTS}

After meeting the inclusion and exclusion criteria 195 patients with 314 knees were included in the study. $40 \%$ patients were Normal Weight, $56.5 \%$ patients were Overweight and $3.5 \%$ patients were obese (Table 2). Mean and Standard deviation of VAS score is $6.63 \pm 1.149$ and BMI is $25.93 \pm 1.98$. There was no significant correlation between BMI and VAS score (Table 3 ).

Table 1: Demographic data.

\begin{tabular}{|lll|}
\hline Characteristics & Number & Percentage (\%) \\
\hline Gender & & 47 \\
\hline Male & 91 & 53 \\
\hline Female & 104 & \\
\hline Age group & & 37.9 \\
\hline $40-50$ & 74 & 34.4 \\
\hline $51-60$ & 67 & 25.1 \\
\hline $61-70$ & 49 & 2.6 \\
\hline $71-80$ & 5 & \\
\hline Mean age & 54.75 & \\
\hline Mean BMI & 25.865 & \\
\hline Total cases & 195 & \\
\hline
\end{tabular}

Table 2: Body mass index wise analysis of sample.

\begin{tabular}{|llllll|}
\hline & & Frequency & Percent & Valid percent & Cumulative percent \\
\hline \multirow{4}{*}{ Valid } & Normal & 78 & 40 & 40 & 40 \\
\cline { 2 - 6 } & Over weight & 110 & 56.5 & 56.5 & 96.5 \\
\cline { 2 - 6 } & Obese & 7 & 3.5 & 3.5 & 100.0 \\
\cline { 2 - 6 } & Total & 195 & 100.0 & 100.0 & \\
\hline
\end{tabular}

Table 3: Correlation between BMI and VAS.

\begin{tabular}{|llll|}
\hline \multirow{4}{*}{ VAS score } & \multicolumn{1}{l}{ Pearson correlation } & 1 & BMI \\
\cline { 2 - 4 } & Sig. (2-tailed) & & .054 \\
\cline { 2 - 4 } & $\mathrm{N}$ & 314 & .344 \\
\hline \multirow{3}{*}{ BMI } & Pearson correlation & .054 & 314 \\
\cline { 2 - 4 } & Sig. (2-tailed) & .344 & 1 \\
\cline { 2 - 4 } & $\mathrm{N}$ & 314 & 314 \\
\hline
\end{tabular}

\section{Correlation between angles and VAS score}

JOA and mTFA were had statistically significant weak positive correlation with VAS (knee pain) score, (r$0.281)(\mathrm{p}-0.000)$ and $(\mathrm{r}-0.236)(\mathrm{p}-0.000)$ respectively. The rest of the angles were not had statistically significant correlation with VAS score.

\section{Correlation between different angles}

Q angle had statistically significant weak negative correlation with mTFA and JOA, (r: -0.162) (p-0.004) and (r-0.282) (p-0.000) respectively. The mTFA had statistically significant weak positive correlation with all 
the other angles. The mean and standard deviation of $\mathrm{m}$ LDFA, $m$ MDFA, $m$ LPTA, $m$ MPTA, $m$ TFA, anatomical TFA and JOA Q-angle demonstrated in Table 4 and 5 .

Table 4: Descriptive Statistics of different angles.

\begin{tabular}{|llll|}
\hline In degrees & Mean & Std. deviation & N \\
\hline VAS score & 6.63 & 1.149 & 314 \\
\hline m LDFA & 88.86 & 2.739 & 314 \\
\hline m MDFA & 91.24 & 2.750 & 314 \\
\hline m LPTA & 95.05 & 2.974 & 314 \\
\hline m MPTA & 84.96 & 3.008 & 314 \\
\hline m TFA & 5.96 & 4.014 & 314 \\
\hline anatomical TFA & 4.55 & 3.674 & 314 \\
\hline JOA & 2.37 & 1.887 & 314 \\
\hline Q-angle & 14.68 & 1.777 & 314 \\
\hline
\end{tabular}

Table 5: Correlation matrix between different angles of knee alignment and VAS score.

\begin{tabular}{|c|c|c|c|c|c|c|c|c|c|c|}
\hline & & $\begin{array}{l}\text { VAS } \\
\text { score }\end{array}$ & $\begin{array}{c}\text { m } \\
\text { LDFA }\end{array}$ & $\begin{array}{l}\text { m } \\
\text { MDFA }\end{array}$ & $\begin{array}{l}\text { m } \\
\text { LPTA }\end{array}$ & $\begin{array}{l}\text { m } \\
\text { MPTA }\end{array}$ & m TFA & a TFA & JOA & Q-angle \\
\hline \multirow{3}{*}{$\begin{array}{l}\text { VAS } \\
\text { score }\end{array}$} & Pearson correlation & 1 & 0.091 & -0.085 & 0.053 & -0.054 & $0.236^{* *}$ & 0.085 & $0.281^{* *}$ & -0.072 \\
\hline & Sig. (2-tailed) & & 0.109 & 0.131 & 0.352 & 0.342 & 0.000 & 0.135 & 0.000 & 0.201 \\
\hline & $\mathrm{N}$ & 314 & 314 & 314 & 314 & 314 & 314 & 314 & 314 & 314 \\
\hline \multirow{3}{*}{$\stackrel{\text { m }}{\text { LDFA }}$} & Pearson correlation & 0.091 & 1 & $-0.949^{* *}$ & $0.241^{* *}$ & $-0.241^{* *}$ & $0.478^{* * *}$ & 0.098 & -0.056 & -0.022 \\
\hline & Sig. (2-tailed) & 0.109 & & 0.000 & 0.000 & 0.000 & 0.000 & 0.084 & 0.323 & 0.699 \\
\hline & $\mathrm{N}$ & 314 & 314 & 314 & 314 & 314 & 314 & 314 & 314 & 314 \\
\hline \multirow{3}{*}{$\begin{array}{l}\mathbf{m} \\
\text { MDF } \\
\text { A }\end{array}$} & Pearson correlation & -0.085 & $-0.949^{* *}$ & 1 & $-0.260^{* *}$ & $0.260^{* *}$ & $-0.512^{* *}$ & $-0.121^{*}$ & 0.045 & 0.041 \\
\hline & Sig. (2-tailed) & 0.131 & 0.000 & & 0.000 & 0.000 & 0.000 & 0.032 & 0.422 & 0.470 \\
\hline & $\mathrm{N}$ & 314 & 314 & 314 & 314 & 314 & 314 & 314 & 314 & 314 \\
\hline \multirow{3}{*}{$\begin{array}{l}\text { m } \\
\text { LPTA }\end{array}$} & Pearson correlation & 0.053 & $0.241^{* *}$ & $-0.260^{* *}$ & 1 & $-0.998^{* *}$ & $0.529^{* *}$ & $0.329^{* * *}$ & -0.006 & $-0.182^{* *}$ \\
\hline & Sig. (2-tailed) & 0.352 & 0.000 & 0.000 & & 0.000 & 0.000 & 0.000 & 0.918 & 0.001 \\
\hline & $\mathrm{N}$ & 314 & 314 & 314 & 314 & 314 & 314 & 314 & 314 & 314 \\
\hline \multirow{3}{*}{$\begin{array}{l}\text { m } \\
\text { MPTA }\end{array}$} & Pearson correlation & -0.054 & $-0.241^{* *}$ & $0.260^{* *}$ & $-0.998^{* *}$ & 1 & $-0.521^{* *}$ & $-0.326^{* *}$ & 0.007 & $0.178^{* *}$ \\
\hline & Sig. (2-tailed) & 0.342 & 0.000 & 0.000 & 0.000 & & 0.000 & 0.000 & 0.904 & 0.001 \\
\hline & $\mathrm{N}$ & 314 & 314 & 314 & 314 & 314 & 314 & 314 & 314 & 314 \\
\hline \multirow{3}{*}{$\begin{array}{l}\text { m } \\
\text { TFA }\end{array}$} & Pearson correlation & $0.236^{* *}$ & $0.478^{* * *}$ & $-0.512^{* *}$ & $0.529^{* * *}$ & $-0.521^{* *}$ & 1 & $0.515^{* *}$ & $0.364^{* *}$ & $-0.162^{* * *}$ \\
\hline & Sig. (2-tailed) & 0.000 & 0.000 & 0.000 & 0.000 & 0.000 & & 0.000 & 0.000 & 0.004 \\
\hline & $\mathrm{N}$ & 314 & 314 & 314 & 314 & 314 & 314 & 314 & 314 & 314 \\
\hline \multirow{3}{*}{ a TFA } & Pearson correlation & 0.085 & 0.098 & $-0.121^{*}$ & $0.329^{* *}$ & $-0.326^{* *}$ & $0.515^{* *}$ & 1 & $0.202^{* *}$ & -0.109 \\
\hline & Sig. (2-tailed) & 0.135 & 0.084 & 0.032 & 0.000 & 0.000 & 0.000 & & 0.000 & 0.054 \\
\hline & $\mathrm{N}$ & 314 & 314 & 314 & 314 & 314 & 314 & 314 & 314 & 314 \\
\hline \multirow{3}{*}{ JOA } & Pearson correlation & $0.281^{* *}$ & -0.056 & 0.045 & -0.006 & 0.007 & $0.364^{* *}$ & $0.202^{* *}$ & 1 & $-0.282^{* *}$ \\
\hline & Sig. (2-tailed) & 0.000 & 0.323 & 0.422 & 0.918 & 0.904 & 0.000 & 0.000 & & 0.000 \\
\hline & $\mathrm{N}$ & 314 & 314 & 314 & 314 & 314 & 314 & 314 & 314 & 314 \\
\hline \multirow{3}{*}{$\begin{array}{l}\text { Q- } \\
\text { angle }\end{array}$} & Pearson correlation & -0.072 & -0.022 & 0.041 & $-0.182^{* *}$ & $0.178^{* *}$ & $-0.162^{* *}$ & -0.109 & $-0.282^{* *}$ & 1 \\
\hline & Sig. (2-tailed) & 0.201 & 0.699 & 0.470 & 0.001 & 0.001 & 0.004 & 0.054 & 0.000 & \\
\hline & $\mathrm{N}$ & 314 & 314 & 314 & 314 & 314 & 314 & 314 & 314 & 314 \\
\hline
\end{tabular}

**. Correlation is significant at the 0.01 level (2-tailed).*. Correlation is significant at the 0.05 level (2-tailed).

\section{DISCUSSION}

The incidence and progression of OA knee are multifactorial. Alignment plays the pivotal role in the etiology of OA. Frontal plane knee alignment is one of the important factors that is responsible for variations in the forces directed at the knee. The risk factors for progression of knee OA, are obesity, quadriceps strength, laxity, and the stage of the disease. These factors influence the malalignment as well. The mean age, sample size, and gender distribution of the patients across published studies were similar to that of our study. ${ }^{18,19}$ The mean \pm SD age of the patients in our study was $54.6 \pm 8.9$ years. In our study, the mean age was less compared to other studies $(64 \pm 11.1,64 \pm 10.8$ and $66.6 \pm 9.2$ years for Sharma et al, Cerejo et al and Felson 
et al respectively) reflecting the onset of disease early in the study population. ${ }^{6,18,20}$ The present study investigated 195 patients (314 knees) with degenerative knee pain, of which $104(53 \%)$ were females. More females with knee pain in our study reflect that early $\mathrm{OA}$ is common in women than men. The number of knees (women) with degenerative knee pain in studies by Sharma et al, Cerejo et al, Evcik et al and Felson et al was 230 (75\%), 230 $(75 \%), 35(77 \%)$ and $270(40 \%)$ respectively. ${ }^{18,20,21}$

Knee malalignment and obesity are risk factors for the incidence and progression of knee OA due to increased joint loading. ${ }^{6}$ We found the mean \pm SD BMI in our study to be $25.86 \pm 1.96$. The above findings comparable to other studies. ${ }^{13,20}$ In our study $56.5 \%$ of patients were overweight (BMI=25-29.9). There was no correlation between BMI and VAS score $(r=0.054$ and $\mathrm{p}=0.344)$. This result was comparable to a similar study done by Evcik et al $(\mathrm{r}=0.47, \mathrm{p}<0.05){ }^{21}$

The Q-angle is used for evaluating patients with knee diseases, especially patellofemoral pain syndrome (PFPS). The static alignment of the lower limb in the frontal plane affects the Q-angle. In our study mean \pm SD Q-angle in patients was $14.68 \pm 1.77$. Q angle had weak negative correlation with mTFA, $(\mathrm{r}=-0.162)(\mathrm{p}=0.004)$ and similarly with JOA $(\mathrm{r}=-0.282)(\mathrm{p}=0.000)$.

There was no correlation between the Q-angle and LDFA $(\mathrm{r}=0.001, \mathrm{p}=0.99)$, and weak positive correlation with MPTA ( $\mathrm{r}=-0.051, \mathrm{p}=0.64)$ in our study. The above finding infers a correlation between the Q-angle and alignment.

A study by Kaya et al.found that there was no correlation between the Q-angle and LDFA ( $\mathrm{r}=0.001, \mathrm{p}=0.99)$, and MPTA $(\mathrm{r}=-0.051, \mathrm{p}=0.64){ }^{22}$ There was no correlation between the Q-angle and lower extremity malalignment. The Q-angle did not present any significant correlation with severity of knee pain $(\mathrm{r}=-0.072 ; \mathrm{p}=0.201)$.

Q angle is a clinical parameter which measured on the patient without a radiograph. It will act as a useful tool in assessing frontal plane alignment because it had statistically significant negative correlation with mTFA and JOA which are affecting the alignment most.

Measurements of lower extremity alignment have considerable influence on decision making and assessing outcomes of the various operative procedures like high tibial osteotomy and total knee replacement. In our study, the frontal plane alignment of the knee was measured by using mechanical axis on full-limb radiographs. The method described above using mechanical axis is the gold standard method for assessing the knee alignment. It has frequently been used in previous studies with similar purposes. ${ }^{6,12,20}$ There are practical and economic limitations that restrict the use of full-limb radiography for assessing mechanical alignment of the knee. Anatomical alignment of the knee measured by standard knee radiography is still widely used as a proxy for mechanical alignment of the knee. Although assessing the tibiofemoral anatomical angle from standardized short knee radiographs has been shown to correlate reliably to the full-length hip-knee axis in numerous studies with Pearson's $r$ values referenced at 0.65 and above, the method is still subject to some debate. ${ }^{9}$ Reported differences between mechanical and anatomical alignments of the knee show wide variations. If variations between mechanical and anatomical alignments of the knee are considerable, interpretations of epidemiologic and clinical studies based on the anatomical alignment of the knee on standard knee radiographs are likely to be inaccurate. ${ }^{19,23}$

Absolute conclusions can't be drawn from our findings because this is a cross-sectional observation. The presumption that radiographic osteoarthritis is a condition with a linear course of deterioration in radio morphological severity. It does suggest that the marked shift in alignment in either direction in osteoarthritic knees is a consequence of the disease rather than a precursor. Because this shift is significantly linearly related to the observed severity of the condition. This point is also in part comparable to a study performed by Sharma et al. ${ }^{6}$ Here, baseline alignment of diagnosed primary OA was assessed and followed up after 18 months, and it was shown that in primary knee OA varus alignment increased the risk of medial OA progression and that valgus alignment increased the risk of lateral OA progression. Measurement, any angles in short knee radiographs are, however, well correlated in experimental studies, only surrogates to measurements made in full limb images. This is a strength in our study as well as in any detailing the angles in standard clinical knee radiographs. We measured the different orientation angles around the knee in patients. Means \pm SD of mLDFA, mMPTA, mTFA, aTFA and JOA were $88.86 \pm 2.73, \quad 84.96 \pm 3.00, \quad 5.96 \pm 4.01, \quad 4.55 \pm 3.67$, and $2.37 \pm 1.88$.

The various angles like mLDFA, mMDFA, mLPTA, mMPTA, mTFA, aTFA, and JOA affect the tibiofemoral alignment and out of which mTFA was considered as the gold standard proven in studies done by Paley et al. ${ }^{24}$ In our study mTFA had statistically significant weak positive correlation with all the angles. These data are comparable with several similar studies. ${ }^{6,13,20}$

Different orientation angles around the knee which were measurements of frontal plane alignment correlated with VAS score (knee pain). JOA and mTFA were had statistically significant weak positive correlation with VAS (knee pain) score, $(\mathrm{r}=0.281) \quad(\mathrm{p}=0.000)$ and $(\mathrm{r}=0.236)(\mathrm{p}=0.000)$ respectively. The rest of the angles were not had statistically significant correlation with VAS score. The above findings reflects that JOA and mTFA which will define the alignment correlate with knee pain. These results were comparable with a similar study by Evcik et al $(\mathrm{r}=0.47)(\mathrm{p}<0.05){ }^{21}$ 


\section{CONCLUSION}

In our study, we found that Frontal plane tibiofemoral alignment correlates with knee pain in patients with early OA. The mTFA may be considered one important criterion in designing treatment and planning surgery for patients with early osteoarthritis. The $\mathrm{Q}$ angle will act as a good alternative clinical tool in assessing frontal plane alignment.

Funding: No funding sources

Conflict of interest: None declared

Ethical approval: The study was approved by the institutional ethics committee

\section{REFERENCES}

1. Cooke TDV, Sled EA, Scudamore RA. Frontal plane knee alignment: a call for standardized measurement. J Rheumatol. 2007;34:1796.

2. Cicuttini F, Wluka A, Hankin J, Wang Y. Longitudinal study of the relationship between knee angle and tibiofemoral cartilage volume in subjects with knee osteoarthritis. Rheumatology. 2004;43:321-4.

3. Mukhopadhyay S, Mukhopadhyay K, Acharyya B. Clinico-radiological assessment of normal variation of alignment of femur and tibia in different age groups in Indian ethnic population. Internet $\mathrm{J}$ Orthop Surg. 2009;15(1):1-5.

4. Chapple CM, Nicholson H, Baxter GD, Abbott JH. Patient characteristics that predict progression of knee osteoarthritis: a systematic review of prognostic studies. Arthritis Care Research. 2011;63:1115-25.

5. Ding C, Garnero P, Cicuttini F, Scott F, Cooley H, Jones G. Knee cartilage defects: association with early radiographic osteoarthritis, decreased cartilage volume, increased joint surface area and type II collagen breakdown. Osteoarthritis Cartilage. 2005;13:198-205.

6. Sharma L, Song J, Felson DT, Shamiyeh E, Dunlop DD. The role of knee alignment in disease progression and functional decline in knee osteoarthritis. JAMA. 2001;286:188-95.

7. Teichtahl A, Wluka A, Cicuttini FM. Frontal plane knee alignment is associated with a longitudinal reduction in patella cartilage volume in people with knee osteoarthritis. Osteoarthritis Cartilage. 2008; 16:851-4.

8. Brouwer G, van Tol AW, Bergink AP, Belo JN, Bernsen RM, Reijman $M$, et al. Association between valgus and varus alignment and the development and progression of radiographic osteoarthritis of the knee. Arthritis Rheum. 2007;56(4):1204-11.

9. Tetsworth K, Paley D. Malalignment and degenerative arthropathy. Orthop Clin North Am. 1994;25:367-78.
10. Tanamas S, Hanna FS, Cicuttini FM, Wluka AE, Berry P, Urquhart DM. Does knee malalignment increase the risk of development and progression of knee osteoarthritis? A systematic review. Arthritis Rheum. 2009;61(4):459-67.

11. Cooke T, Li J, Scudamore RA. Radiographic assessment of bony contributions to knee deformity. Orthop Clin North Am. 1994;25:387-93.

12. Moreland JR, Bassett L, Hanker G. Radiographic analysis of the axial alignment of the lower extremity. JBJS. 1987;69:745-9.

13. Cerejo R, Song J, Dunlop D, Sharma L. The Influence Of Alignment On Risk Of Knee Osteoarthritis Progression According To Baseline Stage Of Disease. Arthritis Rheum. 2001;44:S140.

14. Altman R, Asch E, Bloch D, Bole G, Borenstein D, Brandt $\mathrm{K}$, et al. Development of criteria for the classification and reporting of osteoarthritis: classification of osteoarthritis of the knee. Arthritis Rheumatol. 1986;29:1039-49.

15. Altman RD. Classification of disease: osteoarthritis. in Seminars in Arthritis Rheum. Elsevier. 1991;20:40-7.

16. Reijman M, Pols HA, Bergink AP, Hazes JM, Belo JN, Lievense AM, et al. Body mass index associated with onset and progression of osteoarthritis of the knee but not of the hip: the Rotterdam Study. Ann Rheum Dis. 2007;66(2):158-62.

17. Kellgren J, Lawrence J. Osteo-arthrosis and disk degeneration in an urban population. Ann Rheum Dis. 1958;17:388.

18. Cerejo R, Dunlop DD, Channin D, Song J, Sharma L. The influence of alignment on risk of knee osteoarthritis progression according to baseline stage of disease. Arthritis Rheumatol. 2002;46:2632-6.

19. Hunter DJ, Le Graverand, M.-P.H, Eckstein F. Radiologic markers of osteoarthritis progression. Current Opinion Rheumatol. 2009;21:110-7.

20. Felson DT, Goggins J, Niu J, Zhang Y, Hunter DJ. The effect of body weight on progression of knee osteoarthritis is dependent on alignment. Arthritis Rheumatol. 2004;50:3904-9.

21. Evcik D, Kuru I, Maralcan G, Evcik E. Relationship between the mechanical and anatomic axis and orientation angles of the knee joint and functional capacity of patients with osteoarthritis. Acta Orthop et Traumatologica Turcica. 2006;40:38-43.

22. Kaya D, Doral MN. Is there any relationship between Q-angle and lower extremity malalignment. Acta Orthop Traumatol Turc. 2012;46:416-9.

23. Hunter DJ, Zhang Y, Niu J, Tu X, Amin S, Goggins $\mathrm{J}$, et al. Structural factors associated with malalignment in knee osteoarthritis: the Boston osteoarthritis knee study. J Rheumatol. 2005;32:2192-9.

24. Paley D, Pfeil J. Principles of deformity correction around the knee. Der Orthopade. 2000;29:18-38.

Cite this article as: Rao MK, Reddy PLN. Frontal plane tibio-femoral alignment and its association with knee pain among patients with early osteoarthritis of the knee in a population presenting to a tertiary hospital in South India. Int J Res Orthop 2018;4:79-85. 